Check for updates

Cite this: Phys. Chem. Chem. Phys., 2018, 20, 16294

DOI: $10.1039 / c 8 c p 91772 h$

rsc.li/pccp

\section{Retraction: On the enzymatic activity of catalase: an iron L-edge X-ray absorption study of the active centre}

\author{
Nora Bergmann, ${ }^{a}$ Sébastien Bonhommeau, ${ }^{\text {b Kathrin M. Lange, }{ }^{c} \text { Stefanie M. Greil, }}{ }^{c}$ \\ Stefan Eisebitt, ${ }^{c d}$ Frank de Groot, ${ }^{e}$ Majed Chergui ${ }^{*}{ }^{f}$ and Emad F. Aziz ${ }^{* f}$
}

Retraction of 'On the enzymatic activity of catalase: an iron L-edge X-ray absorption study of the active centre' by Nora Bergmann et al., Phys. Chem. Chem. Phys., 2010, 12, 4827-4832.

We the named authors, hereby wholly retract this Physical Chemistry Chemical Physics article due to inconsistencies in the experimental X-ray absorption spectrum of met-haemoglobin (met-Hb) in Fig. 1a of this PCCP paper. As part of an investigation carried out on behalf of Helmholtz-Zentrum für Materialien und Energie GmbH it was concluded that improper data treatment had occurred in this instance.

Fig. 1a should be a reproduction of the X-ray absorption spectrum published in Fig. 1a of ref. 1, as indicated in the main text. However, the L3/L2 intensity ratios are different and the spectral changes in the PCCP paper contain an abrupt intensity jump at $713 \mathrm{eV}$. The spectrum published in our PCCP paper is not consistent with backups of raw data from the beamline computer and it was thus modified while no specific data treatment is mentioned.

In addition, we note that in ref. 1, the sample was described as a $3 \mathrm{mM}$ aqueous solution of "hemoglobin" (Hb) in the main text, while in the supplemental information it was pointed out that "since native $\mathrm{Hb}$ is readily oxidized in air, the preparation is predominantly methemoglobine (>98\%)". The spectrum presented in Fig. 1a of our PCCP paper was referred to as met-Hb, with no description of the preparation of the solution beyond referencing to ref. 1.

Signed: Nora Bergmann, Sébastien Bonhommeau, Kathrin M. Lange, Stefanie M. Greil, Stefan Eisebitt, Frank de Groot, Majed Chergui and Emad F. Aziz, 22nd May 2018.

Retraction endorsed by Katie Lim, Executive Editor, Physical Chemistry Chemical Physics.

\title{
References
}

1 E. F. Aziz, N. Ottosson, S. Bonhommeau, N. Bergmann, W. Eberhardt and M. Chergui, Phys. Rev. Lett., $2009,102,068103$.

\footnotetext{
${ }^{a}$ Max-Delbrück-Center for Molecular Medicine, D-13125 Berlin-Buch, Germany

${ }^{b}$ Institut des Sciences Moléculaires-UMR 5255 CNRS, Université Bordeaux 1, 351 cours de la Libération, 33405 Talence Cedex, France

${ }^{c}$ Helmholtz-Zentrum Berlin für Materialen und Energie c/o BESSY II, Albert-Einstein Strasse 15, 12489 Berlin, Germany. E-mail: Emad.Aziz@helmholtz-berlin.de

${ }^{d}$ Technical University Berlin, Institute for Optics and Atomic Physics ER 1-1 Strasse des 17. Juni 135, 10623 Berlin, Germany

${ }^{e}$ Department of Inorganic Chemistry and Catalysis, Sorbonnelaan 16, 3584 CA Utrecht, The Netherlands

${ }^{f}$ Ecole Polytechnique Fédérale de Lausanne, Laboratoire de Spectroscopie Ultrarapide, Faculté des Sciences de Base, ISIC-BSP, CH-1015 Lausanne-Dorigny, Switzerland. E-mail: Majed.Chergui@epfl.ch
} 